\title{
Brief Analysis on the Decomposition and Reconstruction of Planar Geometry Elements and the Originality of Garment in Stereo Type
}

\author{
Jun Liang \\ College of Arts \\ Northeast Dianli University \\ Jilin, China
}

\author{
Jiachen Zhu \\ Northeast Dianli University \\ Jinlin, China
}

\begin{abstract}
Nowadays, with the deepening and strengthening of political, economic and cultural exchanges, personalized demand has also been taken seriously. Planar geometry elements have a wide space to play their roles in garment design because of their concise, bright and rich ornamental characteristics, and they constantly bring new visual experience for people and change people's ideology. This paper first explains briefly the concept of planar geometry element, and elaborates how to use planar geometry elements in the innovative design of garment in stereo type respectively from the two aspects of decomposition and reconstruction of planar geometry elements.
\end{abstract}

Keywords-planar geometry element; decomposition and reconstruction; stereo type of garment

\section{INTRODUCTION}

"Clothes are both a representation of culture and an image of thought", Guo Moruo said. China has always been influenced by Confucianism and Taoism, the aesthetic concept of garment modeling is the planar structure of image. This type of planar modeling makes clothes comfortable but not fit, which shows the Chinese implicit, generous and harmonious ideological culture. However, the west has a tradition of advocating human body, which requires the clothes to meet the needs of the fluctuation curve of human body, and better display the beauty of the human body. The application of planar geometry elements in design has a long history, and they are also widely used in garment design. In recent years, the space sense and abstraction of geometric elements have been favored by many fashion designers, who rely on their strong visual effects to make garment design more stereoscopic and forward-looking. The designers recreated the traditional geometric modeling to present the garment outline and pattern in front of people in a new form. They try to inherit and develop on the basis of traditional geometric modeling, so as to create a new way of expression.

\section{OVERVIEW OF PLANAR GEOMETRY ELEMENTS}

Planar geometry elements come from life, by consciously

Fund program: the "pioneer study of garment culture and fashion" of the program of the humanistic and social science base of the general universities in Jilin province (follow-up study). rearranging, compositing and deforming the objective things and graphics in nature through their own abstract thinking, people manage to create highly general and concise artistic image. These irregular geometries belong to a subject of fractal geometry, ("Fractal" is a term developed by the French mathematician Mandelbrot in 1975 based on the Latin term "Frangere", meaning "broken" or "irregular"), and its study object is the non-negative real number dimensionality, which is a universal existence in nature. It is a science that studies irregularity.

With rapid development of science, people are full of curiosity about the unknown world, and they have an endless interest in the abstract and implicit decoration method of geometry. The primary condition of garment designing in modern garment design is to understand the relation between human body and the stereo space of garment. The form of garment modeling is not monotonous; the designers often create different garment outlines by adding, subtracting and combining basic geometric elements on the basis of the basic garment outline. The application of planar geometry elements in modern garment design often conveys the concept of design by combining the garment outline and texture that have a strong sense of architecture with the change of the garment prototype structure, at first look, it may seems "strange", but after a close observation, you will find its details of design are interesting, which catered to the modern approximate "antimainstream" design concept and expressed people's aversion to the now rigid commercial fashion.

\section{THE DECOMPOSITION AND RECONSTRUCTION OF THE PLANAR GEOMETRY ELEMENTS}

Deconstructivism is a kind of anti-traditional thinking tendency which is produced under the influence of the postmodernism cultural thoughts, and it is essentially the destruction and decomposition of structuralism [1]. Deconstructivism can be interpreted in the literal sense as "decomposition" and "reconstruction", which is to recombine the planar geometry elements decomposed from different angles. In garment design, the following methods are often used to decompose and reconstruct geometry elements, such as folding method, segmentation method, reorganization method and accumulation method etc. 


\section{A. Folding Method}

It refers to conducting a three-dimensional processing through the creases of the planar geometric fabric that was already cut. The folding techniques used in geometric art garment include straight line folding method, curve line folding method and irregular folding method. As shown in figure 1, square and triangular three-dimensional modelings are folded on a planar fabric through a regular folding method, and this kind of new geometric modeling is very refreshing.

\section{B. Segmentation Method}

This is to create new geometric modeling through cutting and segmenting the original basic geometric figure and retaining the necessary parts. Adding the same or different geometric shapes on the garment will make the overall visual effect coordinated and balanced. This method will make the garment of planar geometry elements more interesting, and the destructed and indeterminate geometric figure will make the garment type more dynamic and casual. As shown in figure 2, the circles are cut and segmented according to the geometrical calculation method so as to form a new irregular geometry.

\section{Reorganization Method}

This is to recombine the original geometry figures again after they are segmented and interspersed, in the meantime, combine the same or similar parts together, then adjust according to size, shape, number and position to form new geometric shapes; Another method is to use repetition, interspersing, alternation and other techniques to form new combinations, rhythmically vary the use of the same or different figures, and the garment designed by this method can create a strong sense of beauty. In figure 3, the designer segmented and interspersed the circles, then carried out a free design using multiple circles and irregular geometric figures and retained the needed parts, so as to achieve visual balance, especially after recombination of various colors and shapes, the garment modeling is more interesting and the results of various kinds of outline design are obtained. This makes the planar geometry element of garment has the intense visual impact.

\section{Accumulation Method}

It is to repeatedly stack up and accumulate the cloth by use of cutting characteristic of cloth, and put the regular and irregular shapes together in the forms of stitching and pasting, so as to realize the three-dimensional shape change that has layer feeling and make the garment modeling more exaggerated, then to make it more attractive. As shown in figure 4 , the designer divided a piece of square fabric into many identical semicircles according to the geometric figures, and collaged and accumulated these semicircles layer by layer, so as to achieve stereoscopic modeling. The geometric accumulation brings people powerful visual shock, and better interprets the innovative application of the accumulation method in the garment type.

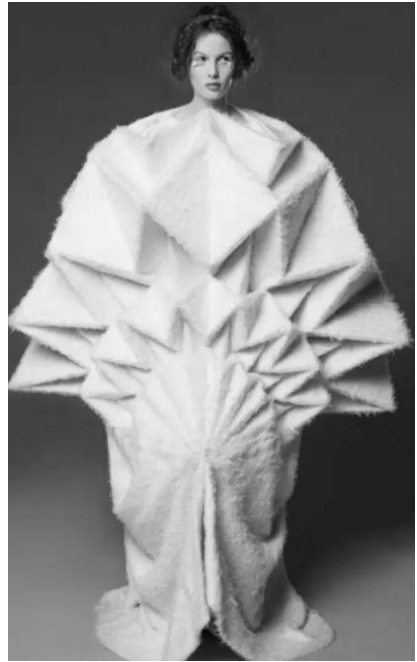

Fig. 1. Garment of folding method.

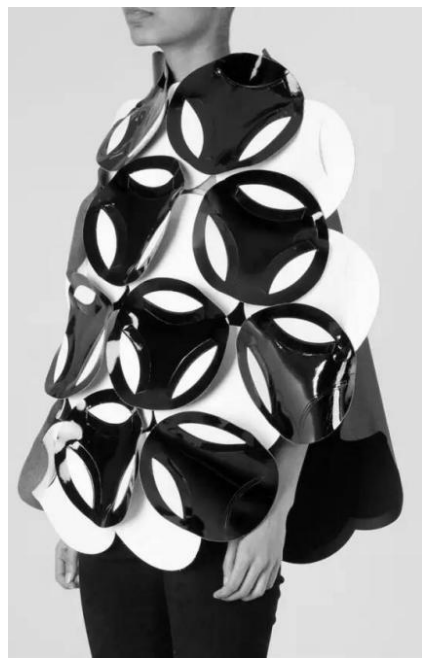

Fig. 2. Garment of segmentation method.

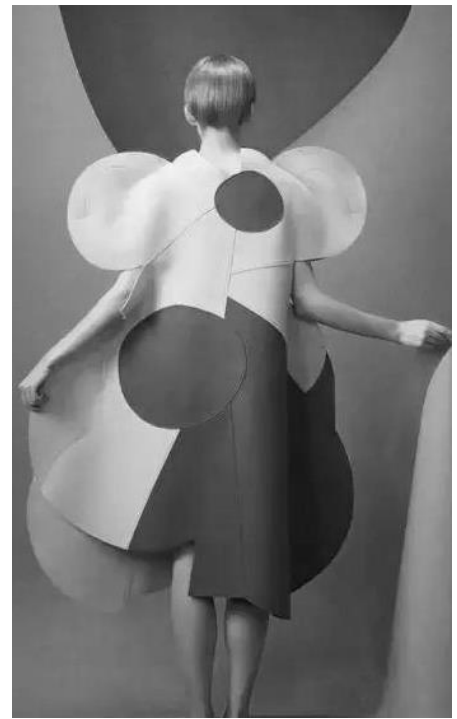

Fig. 3. Garment of reorganization method. 


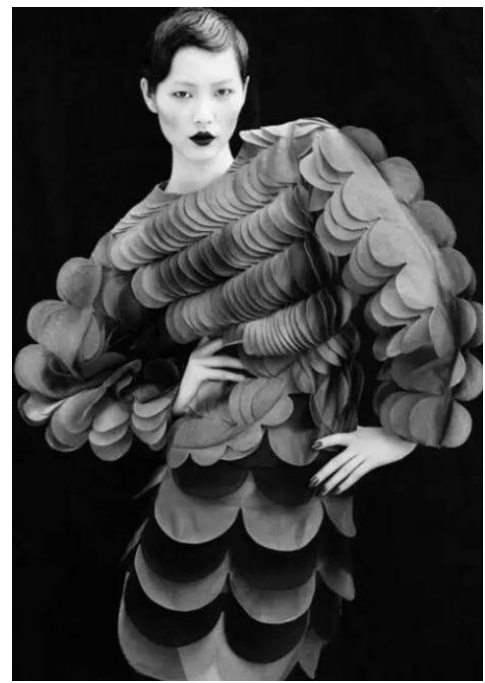

Fig. 4. Garment of accumulation method.

\section{THE GEOMETRIC ELEMENTS AND THE ORIGINALITY OF CONTEMPORARY GARMENT IN STEREO TYPE}

In modern garment design, the stereo type of the garment style and outline is first to be completed, and these threedimensional manifestations need the support of geometrical modeling arts. However, the three-dimensional geometric type is more attractive than the planar geometric pattern. In the broad trend of economic diversification, people are more interested in pursuing the uniqueness of clothing, so the abstraction of geometric elements is more in line with the aesthetic orientation of modern people.

\section{A. The Application of Geometric Elements in the Stereo Modeling of Clothing}

The garment outlines named after geometry include: rectangle, square, circle, ellipse, trapezoid, triangle, etc. Garment design and architecture design both have internal and external space, and the process of garment design is a process of "soft sculpturing of space" by combining all kinds of materials through multiple structural forms, then show colorful geometry types by combining with human body, making the modern stereo modeling of garment more distinctive and richer. For example, the garment in Fig, 5-1, figure 6-1 and Fig. 7-1 are the designs of Ukrainian designer Irina Dzhus in the 2016 spring and summer Lookbook fashion show. Influenced by geometry and architecture, the designer's works are full of geometric details and extremely simple and neat lines. His designs are derived from the vision of minimalism, which creates a simple and unique model through the decomposition and reconstruction of geometric elements. His works are always distinctive tailoring designs and innovative structural designs which have a special geometric architectural aesthetic sense along with a little fun, a little elegance, a little nervousness and industrial texture. Every piece of fabric of the following three garments is given philosophical significance, striving to make each part to be unique through tailoring.

As shown in Fig.5-1, the design method of this garment is to combine the geometric cloth samples by combining the rectangles of different sizes through the straight line folding method, so as to strengthen its layer feeling of threedimensional modeling. The raw edge is fixed on the edge by stitching to make the stereoscopic shapes of the garment more obvious through repetition, rhythm along with the principles. This kind of three-dimensional geometric garment modeling can bring people very strong visual impact, which is simple, unique and conforming to the principles without losing variant. Therefore, it can meet the aesthetic demand of modern people. Fig.5-2 and Fig.5-3 are relatively the paper sample and structure drawing made by the author after studying this garment modeling.

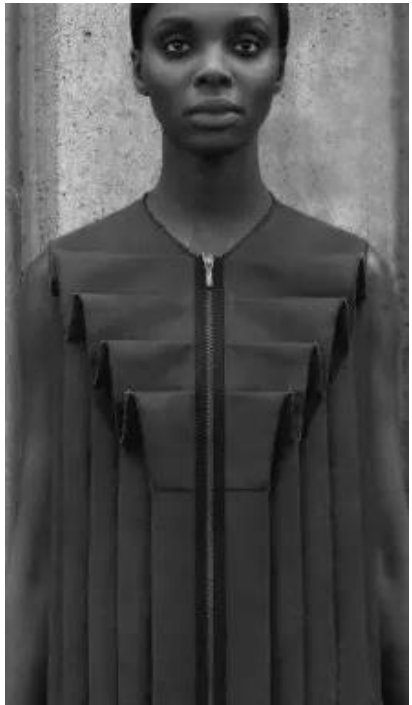

Fig. 5-1 Garment designed by stacking and combination method

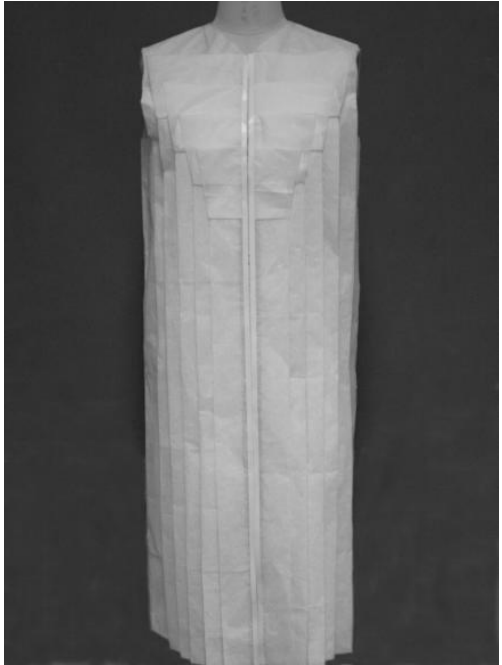

Fig. 5-2 Paper sample of garment designed by stacking and combination method. 


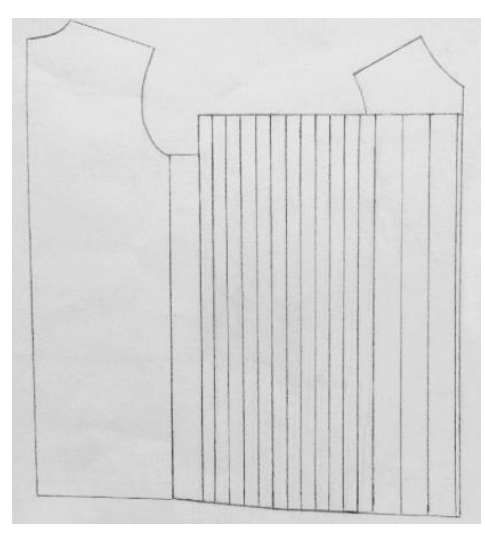

Fig.5-3 Planar drawing of garment designed by stacking and combination method.

In Fig.6-1, the designer recombined the rectangle fabric into irregular geometric modeling after tailoring and folding it. The designer presents the shoulder part using pleat line according to emphasis principle, the aim of which is highlight the characteristic of the geometric subject. The shoulder part is visually widened, and the triangular shape of shoulder shows a sharp and hale feeling, which generates a tough, capable and simple temperament tendency, thus continues Irina Dzhus's consistent simple and industrial design style. This new threedimensional geometry modeling can more satisfy the aesthetic demand of modern people. Figure 6-2 and figure 6-3 are relatively the paper sample and structure drawing made by the author after studying this garment modeling.

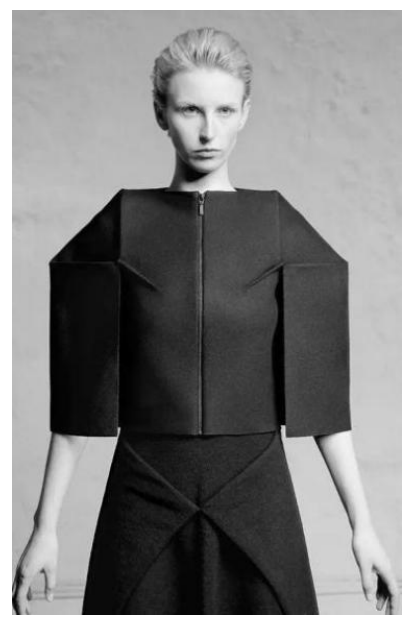

Fig. 6-1 Garment designed by folding and combination method.

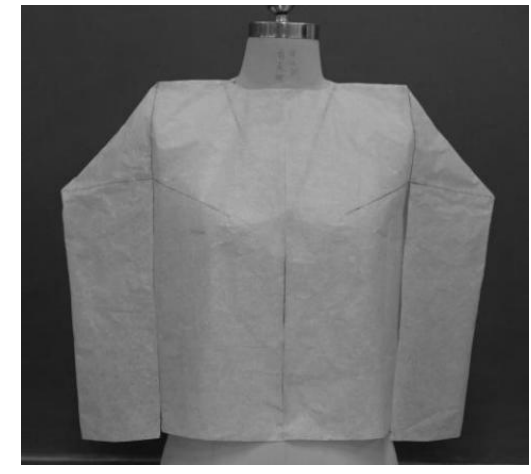

Fig.6-2 Paper sample of garment designed by folding and combination method.

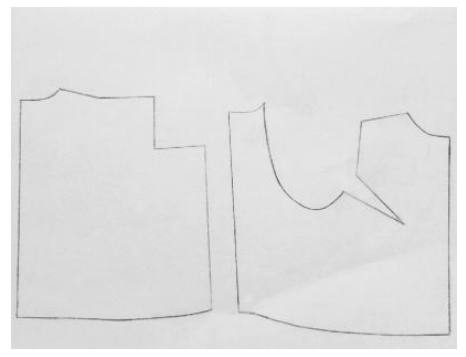

Fig.6-3 Planar drawing of garment designed by folding and combination method.

In Fig.7-1, the designer segmented the rectangular fabric using geometric calculation method according to human body structure, then created irregular geometric shapes through recombination and folding techniques. The pendulous darts and clothing pieces caused by the adjustment of the angle of the shoulder show a sense of dislocation and interspersing, which presents a refreshing geometric modeling. The collar and the placket are fixed on the clothing pieces after folded, which makes the clothing's stereo sense more stronger, so as to create a distinctive visual temptation, which is very refreshing. Fig. 7-2 and Fig. 7-3 are relatively the paper sample and structure drawing made by the author after studying this garment modeling.

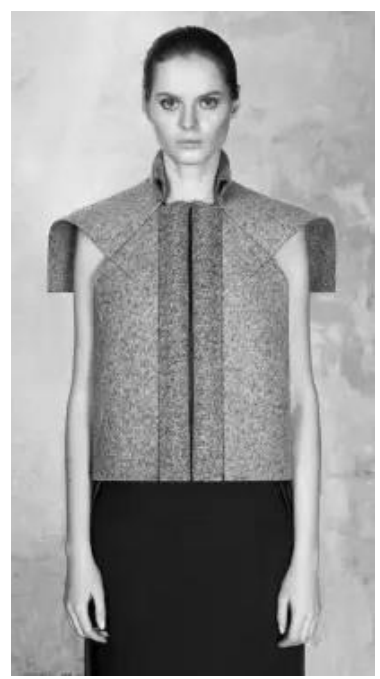

Fig. 7-1 Garment designed by segmentation and recombination method. 


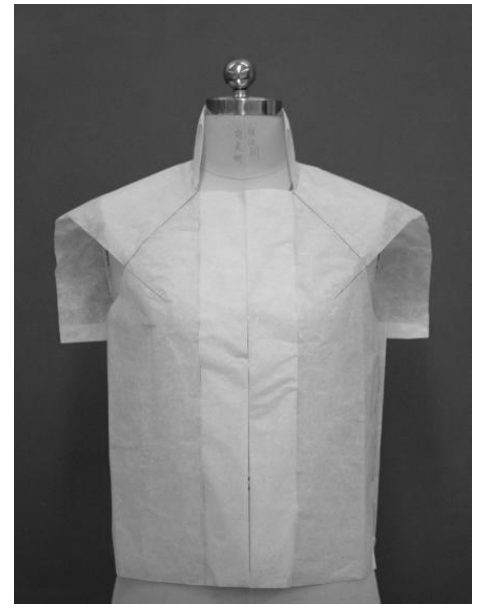

Fig.7-2 Paper sample of garment designed by segmentation and recombination method.

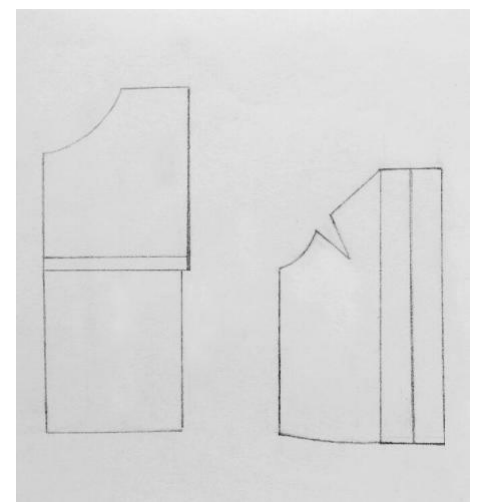

Fig.7-3 Planar drawing of garment designed by segmentation and recombination method.

\section{B. The Adornment Application of Geometric Shape in the Stereo Modeling of Clothing}

The adornment pattern of geometric modeling has a long history in the history of Chinese national culture. in contemporary fashion design, the designer has taken the geometric shapes to the extreme in the garment design, which are also considered as the presentation of unique personality by people. The patterns of three primary colors of red, yellow and blue of "Mondrian garment style" still have great influence on fashion designers now, for example, the application of the interesting collision of bright big color blocks can trigger people's unlimited imagination.

The status of geometric elements in contemporary fashion design is worth approving, and its expression form in garment is also an important question that designers should continue to discuss and study. At present, the application of the planar geometry elements that have the sense of rhythm and order is expanding in the design of women's clothing, and its expression form embodies simplicity and rationality without losing the beauty and elegance of women. The biggest advantage of the geometric shapes is that no matter how simple the style is, it won't seem boring (as shown in figure 8, 9). The integration of the garment design and the geometrical elements, in addition to being able to satisfy the human desire and the creativity of garment modeling, also can increase many unique aesthetic tastes.

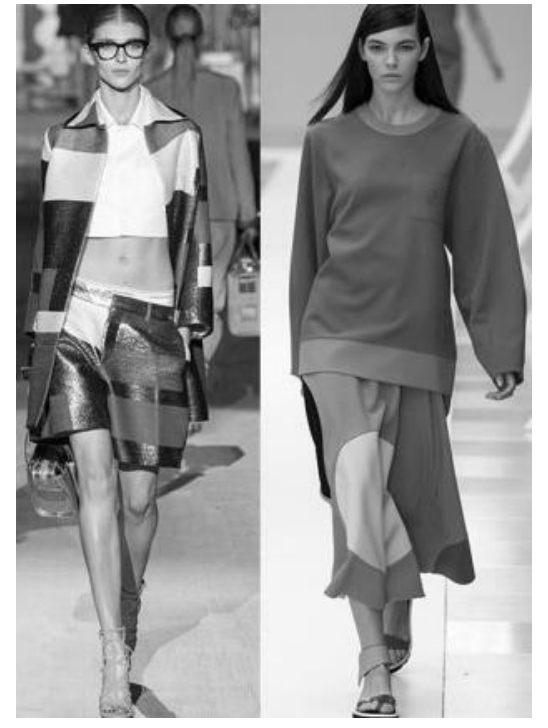

Fig. 8 Garment made by combining irregular geometric patterns.

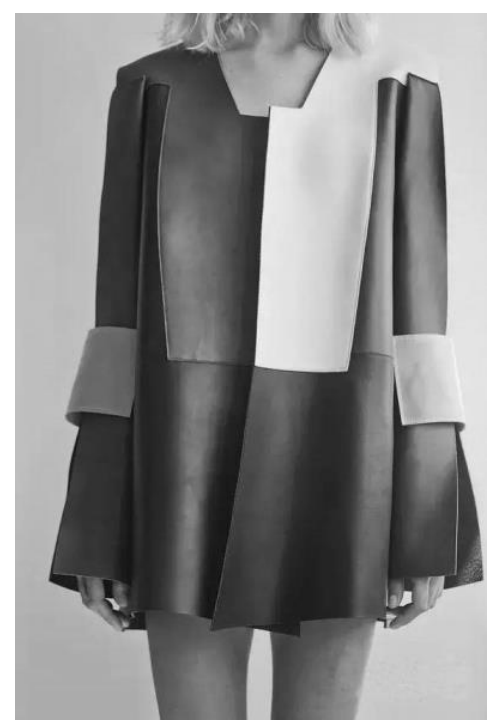

Fig. 9 Garment made by combining geometric patterns.

\section{CONCLUSION}

The creative application of the stereo type of planar geometry elements in garment modeling, and the process of segmenting the planar geometry elements then recomposing them into three-dimensional geometry elements, both highlight the characteristics of the geometrical element clothing, and with the help of it, the designers can create a more new and unique and more avant-garde garment in stereo type. At present, the scholars mainly focus on the study of planar geometry elements and three-dimensional geometry elements, while the future study will be aimed at turning the geometric creative garment into ready-made garment that can be mass produced, which are suitable for with different age groups and 
different price brands. The study and application of planar geometry elements in garment modeling are not only beneficial to broaden the horizon of garment modeling design, but also expand the creative thinking in the garment modeling design, and can help us to understand and master the application methods of the planar geometry elements in the three-dimensional modeling of clothing.

\section{REFERENCES}

[1] Liang Jun. Creativity of garment design - pioneer garment culture and creative design of garment [M]. Beijing: Chemical Industry Press, 2015.

[2] Yang Ming, Sun Jing. The three-dimensional representation of traditional geometric modeling art in modern garment design [J]. Journal of Nanning Vocational and Technical College, 2011 (3). 